\title{
Adjuvante Radiotherapie beim Niedrigrisikomammakarzinom: Langzeitergebnisse der ABCSG-8A-Studie
}

\author{
David Krug ${ }^{1}$ Marciana-Nona Duma² \\ Online publiziert: 16. April 2020 \\ (c) Der/die Autor(en) 2020
}

Hintergrund Der zusätzliche Nutzen der adjuvanten Radiotherapie bei Patientinnen mit Niedrigrisikomammakarzinom im Vergleich zu einer alleinigen endokrinen Therapie nach brusterhaltender Therapie in Bezug auf die Verhinderung eines Lokalrezidivs ist mehrfach belegt durch randomisiert-kontrollierte Studien. Langzeitdaten mit einer Nachbeobachtungszeit von $>10$ Jahren lagen bislang aber nur aus einer Studie vor. Die vorliegende Arbeit präsentiert nun die 10-Jahres-Daten der ABCSG-8A-Studie aus Österreich.

Patienten und Methodik Die ABCSG-8A-Studie ist eine randomisiert-kontrollierte Phase-III-Studie und stellt eine Substudie der ABCSG-8-Studie dar, in der randomisiert 5 Jahre Tamoxifen mit 2 Jahren Tamoxifen, gefolgt von 3 Jahren Anastrozol, verglichen wurde. In die ABCSG8A-Studie eingeschlossen wurden postmenopausale $\mathrm{Pa}-$ tientinnen mit frühem Mammakarzinom: pT1-2 $(<3 \mathrm{~cm})$ pN0, positiver Östrogen- und/oder Progestesteronrezeptorstatus, invasives duktales Karzinom G1-2 oder invasives lobuläres Karzinom. Von 1996 bis 2004 wurden 869 Patientinnen in den Bestrahlungsarm (Bestrahlung der operierten Brust mit ca. 50 Gy, $65 \%$ erhielten eine zusätzliche BoostBestrahlung) oder die alleinige endokrine Therapie randomisiert. Von 519 Patientinnen wurden retrospektiv der HER2-Status und die Ki67-Proliferationsrate erhoben. Anhand dieser Kriterien (HER2-positiv oder Ki67>20\%)

\footnotetext{
Originalpublikation Fastner G, Sedlmayer F, Widder J et al (2020) Endocrine therapy with or without whole breast irradiation in low-risk breast cancer patients after breast-conserving surgery: 10-year results of the Austrian Breast and Colorectal Cancer Study Group 8A trial. Eur J Cancer 127:12-20

$\triangle$ Dr. David Krug david.krug@uksh.de

1 Klinik für Strahlentherapie, Campus Kiel, Universitätsklinikum Schleswig-Holstein, Arnold-Heller-Str. 3, Haus L, 24105 Kiel, Deutschland

2 Jena, Deutschland
}

wurden 8\% der Patientinnen als Hochrisikopatientinnen eingestuft.

Ergebnisse Die mediane Nachbeobachtungszeit betrug 9,9 Jahre. Das lokalrezidivfreie Überleben (LRFS) nach 10 Jahren lag im Bestrahlungsarm bei 97,5\%, verglichen mit 92,5\% nach alleiniger endokriner Therapie (Hazard Ratio 0,27; $p<0,001)$. Auch das krankheitsfreie Überleben (DFS) nach 10 Jahren wurde durch die adjuvante Bestrahlung mit $94,5 \%$ vs. $88,4 \%$ signifikant verbessert $(p=0,02)$. Bezüglich des Gesamtüberlebens (OS) bestand kein signifikanter Unterschied zwischen den beiden Studienarmen. Interessanterweise war der Vorteil im krankheitsfreien Überleben bei Patientinnen, die eine SentinelLymphknoten-Exzision (SLNE) erhalten hatten, deutlich größer $(98,3 \%$ vs. $86,9 \% ; p<0,01)$ als bei Patientinnen, die mit einer Axilladissektion behandelt worden waren (93\% vs. 88,8\%; $p=0,25)$. Neben der Radiotherapie war der einzige weitere signifikante Prognosefaktor hinsichtlich des LRFS das Grading, nicht aber die Tumorbiologie (bei allerdings $<10 \%$ Hochrisikofällen).

Schlussfolgerungen Die Autoren schlussfolgern, dass die adjuvante Bestrahlung der operierten Brust auch bei Mammakarzinomen mit einem niedrigen Risiko das lokalrezidivfreie wie auch das krankheitsfreie Überleben signifikant verbessert. Allerdings überträgt sich dieser Vorteil nicht in ein verlängertes Gesamtüberleben.

\section{Kommentar}

Die adjuvante Radiotherapie der operierten Brust nach brusterhaltender Operation senkt das lokale Rezidivrisiko und verbessert das brustkrebsspezifische und das Gesamtüberleben [1]. Schon in den Metaanalysen der Early Breast Cancer Trialists Cooperative Group wurde aber gezeigt, dass der absolute Vorteil hinsichtlich der Vermeidung eines Lokalrezidivs und damit die ,number needed to treat“ deutlich vom Risikoprofil abhängt und für Niedrigrisiko- 
patientinnen kein Gesamtüberlebensvorteil resultiert [1]. Eine Vielzahl randomisiert-kontrollierter Studien untersuchte daher den Stellenwert der Radiotherapie zusätzlich $\mathrm{zu}$ einer endokrinen Therapie im Vergleich zur alleinigen endokrinen Therapie in diesem Setting (zumeist pT1-2 $[<3 \mathrm{~cm}] \mathrm{pN} 0$, Hormonrezeptor-positiv, G1-2, Alter variabel; [2-7]). In einer Metaanalyse dieser Studien war der Verzicht auf eine adjuvante Radiotherapie mit einem 6,8fach höheren Risiko für ein Lokalrezidiv vergesellschaftet; allerdings bestand kein Nachteil hinsichtlich des Gesamtüberlebens [8]. Bislang hatte nur eine Studie, nämlich die nordamerikanische CALGB-9343-Studie, Langzeitergebnisse nach einer mittleren Nachbeobachtungszeit von 12,6 Jahren präsentiert [5]. Verglichen mit den 5-JahresDaten kam es hier zu einem Anstieg der Lokalrezidivrate von 1 auf $2 \%$ im Bestrahlungsarm, aber von 4 auf $10 \%$ bei alleiniger endokriner Therapie. Somit sank die „number needed to treat" für die Verhinderung eines Lokalrezidivs, allein durch die längere Nachbeobachtungszeit, von 33 auf 13 Patientinnen. Dies war auch in der ABCSG-8AStudie der Fall. Gegenüber den 5-Jahres-Daten [6] stieg die Lokalrezidivrate von 0,5 auf 2,5\% im Bestrahlungsarm an, verglichen mit 5,1 und 7,6\% nach alleiniger endokriner Therapie. Die ,number needed to treat" sank von 22 auf 20 Patientinnen. Eine Verbesserung des krankheitsfreien Überlebens konnte bislang nur in der kanadischen Studie gezeigt werden, die allerdings vielfach kritisiert wurde, da knapp $20 \%$ der Patientinnen einen negativen oder unbekannten Hormonrezeptorstatus und $>30 \%$ einen niedrigen oder unbekannten Differenzierungsgrad aufwiesen [4].

Die Ergebnisse der ABCSG-8A-Studie sind insofern wichtig, weil sie bestätigen, dass der Vorteil der adjuvanten Radiotherapie mit steigender Nachbeobachtungszeit nicht ab-, sondern zunimmt. Relevant ist dies gerade vor dem Hintergrund der steigenden Lebenserwartung: Diese beträgt für eine 1960 geborene Frau z.B. 71,7 Jahre, für ein 2014-2016 geborenes Mädchen 83,2 Jahre und für eine heute 75-jährige Frau 13 weitere Lebensjahre [9]. Ein weiteres Argument zugunsten der adjuvanten Radiotherapie ist die schlechte Therapieadhärenz bezüglich der endokrinen Therapie außerhalb von Studien. Darüber hinaus ist ABCSG 8A die einzige Studie dieser Art, die als Basistherapie eine optimierte endokrine Therapie inklusive eines Aromatasehemmers beinhaltete. Die Beobachtung, dass die DFS-Verbesserung durch die Bestrahlung bei Patientinnen mit SLNE statistische Signifikanz erreichte, nicht aber nach erfolgter Axilladissektion, ist zusätzlich interessant. Die Autoren vermuten, dass dies durch falsch-negative Ergebnisse der SLNE bedingt sein könnte und die okkulten Lymphknotenmetastasen durch die tangentiale Radiotherapie miterfasst und dadurch eliminiert werden [10]. Durch die zunehmende weitere Deeskalation der axillären Chirur- gie, u. a. durch den Verzicht auf die SLNE in der INSEMAStudie, wird diese Annahme prospektiv auf den Prüfstein gestellt werden.

\section{Fazit}

Die Ergebnisse der ABCSG-8A-Studie bestätigen die Effektivität der adjuvanten Radiotherapie bei Patientinnen mit Niedrigrisikomammakarzinom auch bei längerer Nachbeobachtungszeit, wobei der absolute Vorteil mit steigender Nachbeobachtungszeit zunimmt. Ein Verzicht auf die adjuvante Radiotherapie sollte also nur nach einer ausführlichen und individuellen strahlentherapeutischen Beratung erfolgen.

\section{Krug, Kiel, und M.-N. Duma, Jena}

Funding Open Access funding provided by Projekt DEAL.

Interessenkonflikt D. Krug und M.-N. Duma geben an, dass kein Interessenkonflikt besteht.

Open Access Dieser Artikel wird unter der Creative Commons Namensnennung 4.0 International Lizenz veröffentlicht, welche die Nutzung, Vervielfältigung, Bearbeitung, Verbreitung und Wiedergabe in jeglichem Medium und Format erlaubt, sofern Sie den/die ursprünglichen Autor(en) und die Quelle ordnungsgemäß nennen, einen Link zur Creative Commons Lizenz beifügen und angeben, ob Änderungen vorgenommen wurden.

Die in diesem Artikel enthaltenen Bilder und sonstiges Drittmaterial unterliegen ebenfalls der genannten Creative Commons Lizenz, sofern sich aus der Abbildungslegende nichts anderes ergibt. Sofern das betreffende Material nicht unter der genannten Creative Commons Lizenz steht und die betreffende Handlung nicht nach gesetzlichen Vorschriften erlaubt ist, ist für die oben aufgeführten Weiterverwendungen des Materials die Einwilligung des jeweiligen Rechteinhabers einzuholen.

Weitere Details zur Lizenz entnehmen Sie bitte der Lizenzinformation auf http://creativecommons.org/licenses/by/4.0/deed.de.

\section{Literatur}

1. Early Breast Cancer Trialists' Collaborative Group (EBCTCG), Darby S, McGale P et al (2011) Effect of radiotherapy after breastconserving surgery on 10-year recurrence and 15-year breast cancer death: meta-analysis of individual patient data for 10,801 women in 17 randomised trials. Lancet 378:1707-1716. https://doi.org/10. 1016/S0140-6736(11)61629-2

2. Kunkler IH, Williams LJ, Jack WJL et al (2015) Breast-conserving surgery with or without irradiation in women aged 65 years or older with early breast cancer (PRIME II): a randomised controlled trial. Lancet Oncol 16:266-273. https://doi.org/10.1016/S14702045(14)71221-5

3. Blamey RW, Bates T, Chetty U et al (2013) Radiotherapy or tamoxifen after conserving surgery for breast cancers of excellent prognosis: British Association of Surgical Oncology (BASO) II trial. Eur J Cancer 49:1-9. https://doi.org/10.1016/j.ejca.2013.02.031

4. Fyles AW, McCready DR, Manchul LA et al (2004) Tamoxifen with or without breast irradiation in women 50 years of age or older with early breast cancer. N Engl J Med 351:963-970. https://doi.org/10. 1056/NEJMoa040595 
5. Hughes KS, Schnaper LA, Bellon JR et al (2013) Lumpectomy plus tamoxifen with or without irradiation in women age 70 years or older with early breast cancer: long-term follow-up of CALGB 9343. J Clin Oncol 31:2382-2387. https://doi.org/10.1200/JCO.2012.45. 2615

6. Pötter R, Gnant M, Kwasny W et al (2007) Lumpectomy plus tamoxifen or anastrozole with or without whole breast irradiation in women with favorable early breast cancer. Int J Radiat Oncol Biol Phys 68:334-340. https://doi.org/10.1016/j.ijrobp.2006.12.045

7. Tinterri C, Gatzemeier W, Costa A et al (2013) Breast-conservative surgery with and without radiotherapy in patients aged 55-75 years with early-stage breast cancer: a prospective, randomized, multicenter trial analysis after 108 months of median follow-up. Ann Surg Oncol 21:408-415. https://doi.org/10.1245/s10434-013$3233-x$
8. Matuschek C, Bölke E, Haussmann J et al (2017) The benefit of adjuvant radiotherapy after breast conserving surgery in older patients with low risk breast cancer-a meta-analysis of randomized trials. Radiat Oncol 12:60-68. https://doi.org/10.1186/s13014-017-0796$\mathrm{x}$

9. Homepage des Bundesinstituts für Bevölkerungsforschung. https:// www.bib.bund.de/Permalink.html?id=10262290. Zugegriffen: 20. Febr. 2020

10. Borm KJ, Oechsner M, Düsberg M et al (2020) Irradiation of regional lymph node areas in breast cancer-dose evaluation according to the Z0011, AMAROS, EORTC 10981-22023 and MA-20 field design. Radiother Oncol 142:195-201. https://doi.org/10.1016/j. radonc.2019.08.021 